\title{
MORFOLOGÍA, CARACTERIZACIÓN Y DISTRIBUCIÓN GEOGRÁFICA DE BLECHNUM CORDATUM (BLECHNACEAE-PTERIDOPHYTA)
}

\author{
Carmen PRADA ${ }^{1 *}$, Cristina H. ROLLERI ${ }^{2}$ y Lilian PASSARELLI ${ }^{2}$ \\ ${ }^{1}$ Departamento de Biología Vegetal I, Facultad de Ciencias Biológicas, Universidad Complutense, \\ Ciudad Universitaria, 28040 Madrid, España \\ ${ }^{2}$ Laboratorio de Estudios de Anatomía Vegetal Evolutiva y Sistemática (LEAVES), Facultad de \\ Ciencias Naturales y Museo de La Plata, 64 entre 120 y diagonal 113, B1904 DZB, \\ La Plata, Argentina. \\ *Autora para correspondencia: cpm@bio.ucm.es \\ Recibido el de 16 de julio de 2007, aceptado para publicación el 13 de junio de 2008 \\ Publicado "on line" en julio de 2008
}

RESUMEN. Morfología, caracterización y distribución geográfica de Blechnum cordatum (Blechnaceae-Pteridophyta). Blechnum cordatum fue estudiado en especímenes de numerosas localidades de su extensa área de distribución. Crece en Mesoamérica, Antillas, Sudamérica, desde Venezuela y Colombia a Bolivia, SE y centro de Brasil, Paraguay, Argentina, centro y S de Chile e islas de Juan Fernández. Es una especie poco vulnerable, tolerante, con esporófitos grandes, rizomas erectos escamosos, frondas dimorfas con estípites y otros ejes escamosos; láminas estériles lanceoladas a oblongas con pinnas coriáceas, lanceolado-oblongas, finamente denticuladas a aserradas, superficialmente escamosas y pilosas, unidas al raquis por la costa (pecioluladas), con bases cuneadotruncadas a subcordadas o auriculadas y láminas fértiles estrechamente lanceoladas con el tejido vegetativo de las pinnas reducido a la porción de la lámina que lleva el cenosoro continuo e indusio ondulado a eroso. Las venas son simples, geminadas y bifurcadas al azar a distancias variables de la costa y terminan en grandes hidatodos activos, sobresalientes o más o menos planos. Los aeróforos, presentes sólo en la base de las pinnas, pueden faltar. Las esporas son monoletas, con perisporio crestado-reticulado que lleva procesos filiformes y exosporio subliso a granulado. La especie se describe e ilustra en detalle, se actualiza su taxonomía y se comentan sus afinidades con otras especies neotropicales y paleotropicales del género.

Palabras clave. Blechnaceae, Blechnum cordatum, morfología, taxonomía, palinología, distribución geográfica.

ABSTRACT. Morphology, characterization, and geographical distribution of Blechnum cordatum (Blechnaceae-Pteridophyta). Specimens of Blechnum cordatum from localities of its large geographical area were analized. The species grows in Mesoamerica, Antillas and South America, from Venezuela and Colombia to Bolivia, SE and centre of Brazil, Paraguay, Argentina, centre and S of Chile, and

Este trabajo contó con el apoyo de la Agencia Española de Cooperación Internacional (Proyecto AECI A6307/06), de la Universidad Complutense de Madrid, del Consejo Nacional de Investigaciones Científicas y Técnicas de Buenos Aires (CONICET) y del Programa de Incentivos para Docentes Investigadores de la Universidad Nacional de La Plata, Argentina, (Proyecto N 11/434-2007) . 
Juan Fernández Islands. Blechnum cordatum is a tolerant, not vulnerable species, with large sporophytes; erect, scaly rhizomes; dimorphic fronds with scaly stipes and axes; lanceolate sterile laminae with lanceolate to oblong, coriaceae, finely denticulate to serrate, superficially scaly and hairy, attached by costa (peciolulate) pinnae, with cuneate- truncate to subcordate or auriculate bases, and narrowly lanceolate fertile laminae with vegetative tissue of pinnae reduced to the portion which support the undulate to erose indusia and continuous coenosorus. Veins are free, simple, geminate and furcate, the latter ramdomly dividing at different distances from the costa, all ending in large, active hydathodes. Aerophores, located only at the base of pinnae, may be absent. Spores have a cristate-reticulate perispore with filiform, ramified processes, and a smooth to granulate exospore. Based on this study, a new description of Blechnum cordatum, and its taxonomy is presented, along with comments on affinities with other neotropical and paleotropical species of the genus.

Key words. Blechnaceae, Blechnum cordatum, morphology, taxonomy, palynology, geographical distribution.

\section{INTRODUCCIÓN}

Blechnum cordatum (Desv.) Hieron. ha sido tratada por Stolze (1981), Tryon \& Stolze (1993), Kazmircsack (1999), Ramos Giacosa et al. (2004, 2006); Mickel \& Smith (2005), Oliveira Dittrich (2005) y Rolleri \& Prada (2006 a). La mayoría de esos autores hacen referencias a las variaciones morfológicas que presenta la especie.

Tryon \& Tryon (1982) incluyeron a $B$. cordatum en el grupo B. lineatum (Sw.) C. Chr., con otras 15-20 especies poco conocidas, entre ellas $B$. chilense (Kaulf.) Mett., B. chiriquanum (Broadh.) C. Chr., B. costaricense (Broadh.) C. Chr., B. divergens (Kunze) Mett., B. lehmanii Hieron., B. lherminieri (Bory) C. Chr., B. lineatum, $B$. pteropus (Kunze) Mett., B. sprucei C. Chr. y B. varians (Fourn.) C. Chr., todas representadas por plantas grandes, con tallos masivos decumbentes, cortamente reptantes o semiescandentes y frondas dimórficas.

Posteriormente, Tryon \& Stolze (1993) estudiaron material de $B$. cordatum procedente de Perú e incluyeron en su sinonimia a $B$. chilense. En floras, catálogos $\mathrm{u}$ otros trabajos sobre Blechnum en el cono sur (de la Sota, 1977; Rolleri, 1984; Rodríguez Ríos, 1995; Ponce, 1996; Durán, 1997; Arana et al., 2004; Dellucchi, 2006) se hacen referencias casi exclusivamente a $B$. chilense y no se comentan sus afinidades con B. cordatum. Ramos Giacosa et al. (2004, 2006) citaron a B. cordatum como una especie nueva para la flora Argentina.

Moran (1995) estudió material mesoamericano de B. schiedeanum (Schltdl. ex C. Presl) Hieron. (=B. costaricense) y la consideró muy similar a $B$. cordatum. Kazmircsack (1999) sugirió que las especies de Brasil B. macahense Brade, B. raddianum Rosenst., B. regnellianum (Kunze) C. Chr. y $B$. simile Sehnem integrarían un grupo de formas relacionadas con $B$. cordatum, $B$. falciforme (Liebm.) C. Chr. y B. lineatum, todas ellas con fenotipos muy similares y algo variables.

Oliveira Dittrich (2005) incluyó $B$. macahense, $B$. raddianum, $B$. regnellianum y $B$. simile entre los sinónimos de $B$. cordatum, por entender que representan un continuum de formas del mismo taxón junto con $B$. itatiaiense Brade, una especie cuya descripción se basó en un ejemplar de $B$. cordatum, muy abundante en el Parque Nacional de Itatiaia y en general, en las selvas montanas del E-SE de Brasil (Santos Condack, 2006). Oliveira Dittrich (2005), además, segregó del grupo $B$. lineatum a todas las especies con pinnas pecioluladas, indumento abundante en ejes y láminas y aeróforos o bulbillos en el raquis, a las que propuso reunir en el grupo $B$. cordatum, integrado en Brasil también por B. glaziovii H. Christ, B. proliferum Rosenst., B. sprucei 
C. Chr. y B. usterianum (H. Christ) C. Chr. Todas estas especies se caracterizan por su tolerancia ecológica, ya que crecen tanto en selvas montanas y pedemontanas como en bordes de selvas y bosques húmedos o áreas más expuestas.

El objetivo de este estudio fue analizar la variación morfológica de $B$. cordatum en ejemplares procedentes de distintas localidades de su área de distribución, analizar la variación morfológica en relación con la geografía y establecer los rasgos que caracterizan la especie. Se estudiaron los tipos de rizoma, el indumento de los ejes, la división de la lámina, el contorno, textura, tamaño, margen, indumento superficial, venación, modelo epidérmico y estomas de las pinnas estériles, la ontogenia del modelo epidérmico, la morfología interna de los estípites juveniles y adultos en sección transversal y las esporas. Todos estos caracteres, en conjunto, no se habían utilizado anteriormente en las descripciones de la especie.

Los rasgos exomorfológicos y endomorfológicos se compararon en especímenes coleccionados a diferentes latitudes y alturas sobre el nivel del mar, en áreas continentales como selvas bajas, pedemontanas, bosques tropicales y subtropicales, bordes de selvas y en áreas insulares. Como resultado de este análisis se presenta una descripción completa, con la sinonimia actualizada, comentarios sobre relaciones interespecíficas, afinidades, ecología y distribución geográfica de $B$. cordatum, en relación con otras especies americanas y extramericanas de Blechnum.

\section{MATERIAL Y MÉTODO}

Se utilizó material fresco y de herbario. El material de herbario se restauró con una mezcla de butil cellosolve y agua 1:4. Las pinnas se aclararon con hidróxido de sodio al $6 \%$ y se colorearon con azul de toluidina (TBO) $1 \%$ acuoso.

La venación y los modelos epidérmicos se analizaron en pinnas basales, medias y apicales, pero se ilustran sólo los de las pinnas medias. Las dimensiones y densidad de los estomas se tomaron en pinnas medias de todos los ejemplares estudiados; las dimensiones se expresan en largo $\mathrm{x}$ ancho, en $\mu \mathrm{m}$ y la densidad en número de estomas por $\mathrm{mm}^{2}$. Los valores dados representan el promedio de 25 medidas por muestra. Los ejemplares que no han sido estudiados anatómicamente se señalan con asterisco $(*)$ en la lista de material estudiado.

Las muestras de esporas se tomaron de especímenes de todas las localidades en las que se disponía de frondas fértiles. Se midieron con microscopio óptico (diámetro mayor x diámetro menor, en $\mu \mathrm{m}$ ). Los valores dados representan el promedio de 30 medidas por muestra. Para estudios con microscopía electrónica de barrido, las esporas se montaron sin tratamiento, se cubrieron con oro bajo vacío, en un metalizador Jeol JSM 1100. Las fotografías se tomaron con un microscopio electrónico Jeol JFC T100.

Toda la terminología relacionada con el esporófito está en Lellinger (2002), la referente a la epidermis es la que han utilizado las autoras en trabajos pteridológicos previos, como Rolleri \& Prada (2006 b), la relacionada con los estomas se ha tomado de Prabhakar (2003) y la referente a las esporas, con algunas variantes, de Lellinger \& Taylor (1997) y Lellinger (2002).

\section{RESULTADOS}

El rizoma es robusto, rígido, de cortamente rastrero a procumbente en plantas 
jóvenes y erecto, formando un pequeño tronco en plantas adultas. No produce estolones y está cubierto por escamas grandes, concoloras, de color castaño claro, translúcidas.

Las frondas son marcadamente dimórficas, las estériles divergentes, por lo general más cortas que las fértiles, que son erguidas, con los estípites más largos y pinnas lineares con la lámina extremadamente reducida.

Los estípites representan hasta 1/3-1/2 de la longitud total de las frondas. Suelen ser más largos en plantas que crecen en colonias abundantes y más cortos en plantas solitarias o de colonias ralas. Son rígidos en la madurez, adaxialmente acanalados y con abundantes escamas basales similares a las del rizoma, cuya densidad puede variar. Tienen color verde intenso en plantas jóvenes y de castaño a castaño oscuro en plantas maduras; en la base puede tener una coloración pardo-rojiza, rojiza o magenta. En sección transversal se observan una epidermis papilosa con sus paredes externas marcadamente engrosadas y cutinizadas, una zona hipodérmica fibrosa cuyo espesor en capas depende de la edad de las plantas y un tejido interno parenquimático no amiláceo que incluye la estela. Los cortes seriados del estípite de plántulas y plantas de hasta 25 $\mathrm{cm}$ de altura indican que la estela está formada por 3 haces vasculares en los primeros 3-6 cm del estípite de las plántulas (fig. 1 B) y que a unos 10-12 cm de la base, la estela está más fragmentada y presenta 46 haces de diferente tamaño (fig. 1 C-D). Las plantas de $30-35 \mathrm{~cm}$ de altura no tienen más de 6 haces vasculares en el estípite (fig. 1 D). Las plantas adultas más grandes tienen estípites de sección casi romboidal, adaxialmente acanalados, con un surco profundo y abaxialmente carenados. La zona hipodérmica está formada por 12-15 capas de fibras marcadamente lignificadas y la dictiostela tiene 14-16 meristelas incluidas en un área central parenquimática masiva (fig. $1 \mathrm{~F}$ ).

La lámina estéril es de lanceolada a lanceolado-oblonga, imparipinnada (fig. $1 \mathrm{E}$, $\mathrm{G})$, con ápice agudo y base truncada. El color varía de verde glauco a verde intenso y en plantas expuestas puede ser verde amarillento, verde-rojizo o magenta, con láminas de distinto color en la misma planta.

El raquis es escamoso, variablemente papiloso y puede presentar aeróforos en la base de las pinnas. Los aeróforos son oblongos, grandes, sobresalientes, visibles con escaso aumento, particularmente en los ejemplares más australes, pero pueden ser chatos, de contorno elíptico, menos evidentes, como pequeños abultamientos en forma de cabezuela o aguijón bajo en ejemplares de selvas. Están bien desarrollados en los circinos, donde pueden aparecer como protuberancias digitiformes de hasta $2 \mathrm{~mm}$ y tienden a perder prominencia, adoptando el aspecto de pequeñas manchas o hasta concavidades oscuras en plantas adultas.

Las costas son acanaladas, prominentes, adaxialmente trisurcadas y abaxialmente curvas, papilosas y escamosas, con papilas unicelulares, redondeadas, más prominentes que las del raquis y escamas abundantes que se continúan en las venas y, más dispersas, sobre la superficie de la lámina. En sección transversal presentan tres haces vasculares.

La pinna terminal está bien desarrollada, es lanceolada, conforme con las laterales pero siempre más larga, con el ápice agudo y la base cordada ancha. En plántulas y plantas jóvenes la base es algo asimétrica, mientras que en plantas adultas puede ser marcadamente ensanchada y con frecuencia, levemente hendida en uno de los hemifilos (fig. $1 \mathrm{E}, \mathrm{G}$ ).

Las pinnas laterales se presentan con un número variable de pares según las etapas 


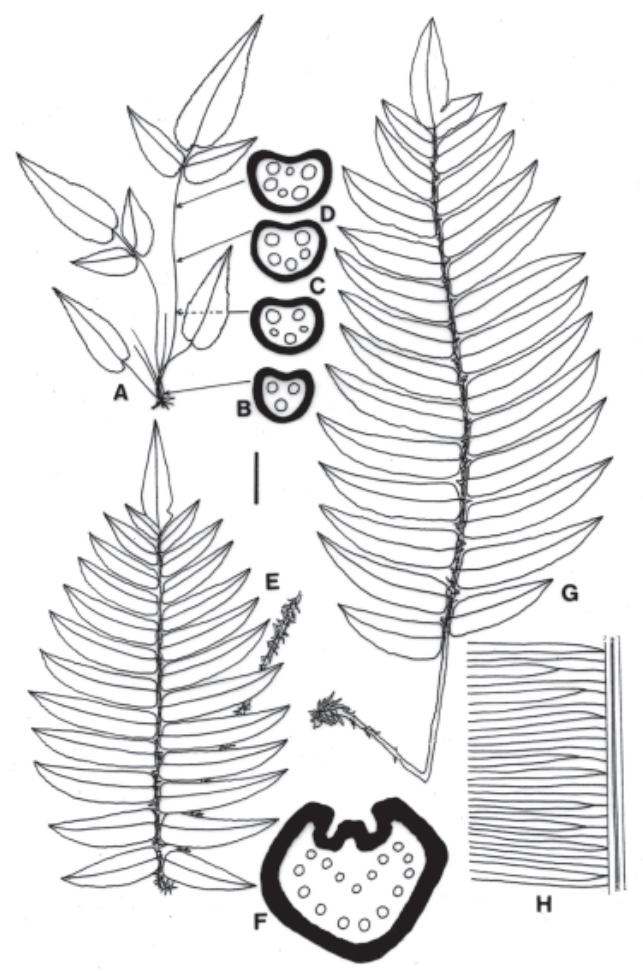

Figura 1. Caracteres de las frondas de $B$. cordatum. A: planta juvenil, de Argentina, Río Negro, Prada $s / n$ (MA). B-D: Secciones transversales del estípite en A, mostrando la fragmentación de la dictiostela original. E: Fronda completa de B. cordatum de Chile, Concepción, Pérez Moreau 22912 (BA). F: Sección transversal de estípite adulto en E. G: Fronda completa de B. cordatum de Brasil, Santa Catarina, Sehnem 68898 (BA). H: Venación en pinnas de $B$. cordatum, con venas simples, geminadas y bifurcadas a diferentes distancias de la costa, casi paralelas, horizontales y próximas. En negro, área hipodérmica fibrosa. Barra $=6 \mathrm{~cm}$ en A, E y G; $0,18 \mathrm{~cm}$ en B-D; $0,8 \mathrm{~cm}$ en F; 0,5 $\mathrm{cm}$ en $\mathrm{H}$. Characters of the fronds of B. cordatum. A: Young plant, from Argentina, Rio Negro, Prada $s / n(M A)$. B-D: Transversal sections of stipe in A, showing the fragmentation of the original dictyostele. E: An entire frond of $B$. cordatum from Chile, Concepción, Pérez Moreau 22912 (BA). F: Transversal section of adult stipe in E. G: An entire frond of $B$. cordatum from Brasil, Santa Catarina, Sehnem 68898 (BA). H: Venation in pinnae of $B$. cordatum, with simple, geminate, and furcate veins, dividing at different distances from the costa, almost parallel, horizontal, and approximate. In black, hypodermal fibrous layers. Bar $=6 \mathrm{~cm}$ in $A, E$ and $G ; 0,18 \mathrm{~cm}$ in $B-D ; 0,8 \mathrm{~cm}$ in $F ; 0,5 \mathrm{~cm}$ in $H$. de crecimiento de las plantas. En las plántulas, cuyas frondas tienen estípites de hasta $22 \mathrm{~cm}$, se hallan 1-3 pinnas (fig. $1 \mathrm{~A}$ ). En plantas pequeñas a medianas (hasta 45 cm) se encuentran 4-7 pares de pinnas, en plantas más grandes (hasta $120 \mathrm{~cm}$ ) hay 1420 pares, mientras que ese número puede elevarse hasta 40 pares en las plantas más grandes. Son de lanceoladas a lanceoladooblongas, de levemente a marcadamente ascendentes, con ápice agudo, a veces prolongado en un extremo aserrado, base simétrica cordada o asimétrica, con el lado basiscópico más redondeado que el acroscópico, que puede ser algo cuneado y hasta auriculado. Están unidas al raquis por una extensión de la costa y son marcadamente pecioluladas en algunos ejemplares. Ocasionalmente, la pinna terminal y el primer par lateral son adnatos. Las pinnas del tercio apical y basal pueden ser casi sésiles pero nunca son totalmente adnatas y la porción basiscópica es auriculada e invariablemente libre, aún cuando la acroscópica sea sésil. La base de la lámina es más o menos abruptamente redondeado-cordada, sin pinnas vestigiales (fig. $1 \mathrm{E}, \mathrm{G}$ ).

La textura de la lámina es herbácea firme en plantas jóvenes o en las que crecen en selvas húmedas, y de subcoriácea a coriácea en plantas muy grandes o que crecen en áreas expuestas o a mayor latitud o altura sobre el nivel del mar. La superficie también puede variar: es opaca, lisa, subpapilosa o papilosa en plantas jóvenes o en las que crecen en selvas húmedas y brillante u opaca, rugosa o estriada en plantas grandes o que crecen expuestas, a mayor latitud o a mayor altura sobre el nivel del mar.

Los márgenes son planos, aserrados y finamente dentados, generalmente con dentículos anchos dirigidos acroscópicamente, muy marcados en el ápice y pueden tornarse revolutos u ondulado- 
repandos en las plantas más grandes y expuestas.

Los aeróforos se encuentran en la base de las pinnas; tienen contornos y formas variables en sus etapas juveniles (circinos) y en sección parecen domos o protuberancias digitiformes con la epidermis formada por células de contorno poligonal y abundantes estomas en formación. Suelen tener color de rojizo a magenta debido a pigmentos antociánicos. Los estomas son predominantemente anomocíticos y las células del tejido subepidérmico se dividen desordenadamente y a veces están coloreadas de castaño, por la presencia de taninos. Los aeróforos adultos muestran una gran actividad de división en estas células, que terminan por formar un tejido laxo relacionado con el intercambio gaseoso a la vez que la epidermis se desgarra formando una estructura con el aspecto de una lenticela angiospérmica, que interrumpe la continuidad de la capa de fibras. Cuando las frondas maduran o alcanzan su máximo desarrollo el parénquima acumulado desordenadamente por debajo de la zona originalmente estomática puede engrosarse y ese proceso cancelaría la función local de aireación. En estas etapas, los aeróforos son menos visibles e incluso pueden formar áreas oscuras parcialmente hundidas y no sobresalientes.

La venación es libre, con venas simples, geminadas y bifurcadas cerca de la costa y a distintas distancias de ésta (fig. $1 \mathrm{H}$ ). La proporción de venas geminadas puede variar en distintos ejemplares. Las venas se ensanchan en la porción terminal finalizando en hidatodos grandes y visibles, poco sobresalientes, ubicados muchas veces en los dentículos marginales que pueden ser anchos $\mathrm{y}$ triangulares.

El indumento está representado por pelos y escamas. Se ha observado la variación siguiente:
En los rizomas y la base del estípite hay escamas lanceoladas, basifijas, largamente atenuadas, de color pardo-amarillento o castaño, generalmente concoloras, diáfanas, claras. Son enteras, aunque ocasionalmente pueden tener largas laceraciones piliformes aisladas en los márgenes (fig. 2 P). Están compuestas por células angostas, hasta 10 veces más largas que anchas y con paredes delgadas (fig. 2 Q).

En los circinos y frondas tenuerales hay pelos y escamas. Los pelos son glandulares, mucilaginíferos, con un cuerpo formado por 2-4 células cilíndricas y una cabezuela grande y redondeada (fig. 2 A-C). Se distribuyen superficialmente y en las áreas de venas, en éstas de manera muy abundante y densamente aglomerados (fig. 2 B). Son parcialmente caducos en el curso del crecimiento y en las frondas en despliegue y adultas están dispersos. Son más abundantes en plantas de áreas selváticas que en plantas de zonas más expuestas. Las escamas están distribuidas abundantemente en la superficie de la lámina, en las costas y el raquis juveniles, densamente entremezcladas con pelos glandulares en los circinos. Son peltadas y tanto en los circinos (fig. $2 \mathrm{D}-\mathrm{F}$ ) como en las plantas en crecimiento o adultas están formadas por pocas células (fig. 2 G$\mathrm{J})$; el cuerpo es irregular, a veces casi estrellado, con brazos divergentes que terminan en células de extremos más o menos agudos (fig. 2 D-K). Parecen ser parcialmente caducas y a medida que las plantas maduran disminuyen en densidad sobre la lámina pero no sobre los ejes.

En los ejes y las láminas adultas también se encuentran escamas y pelos. Las escamas predominan sobre las costas y raquis, donde son abundantes, de color castañoamarillento, peltadas, lanceoladas anchas con procesos laterales agudos de irregulares a piliformes (fig. 2 L-O). En general se continúan también a lo largo de las venas. 


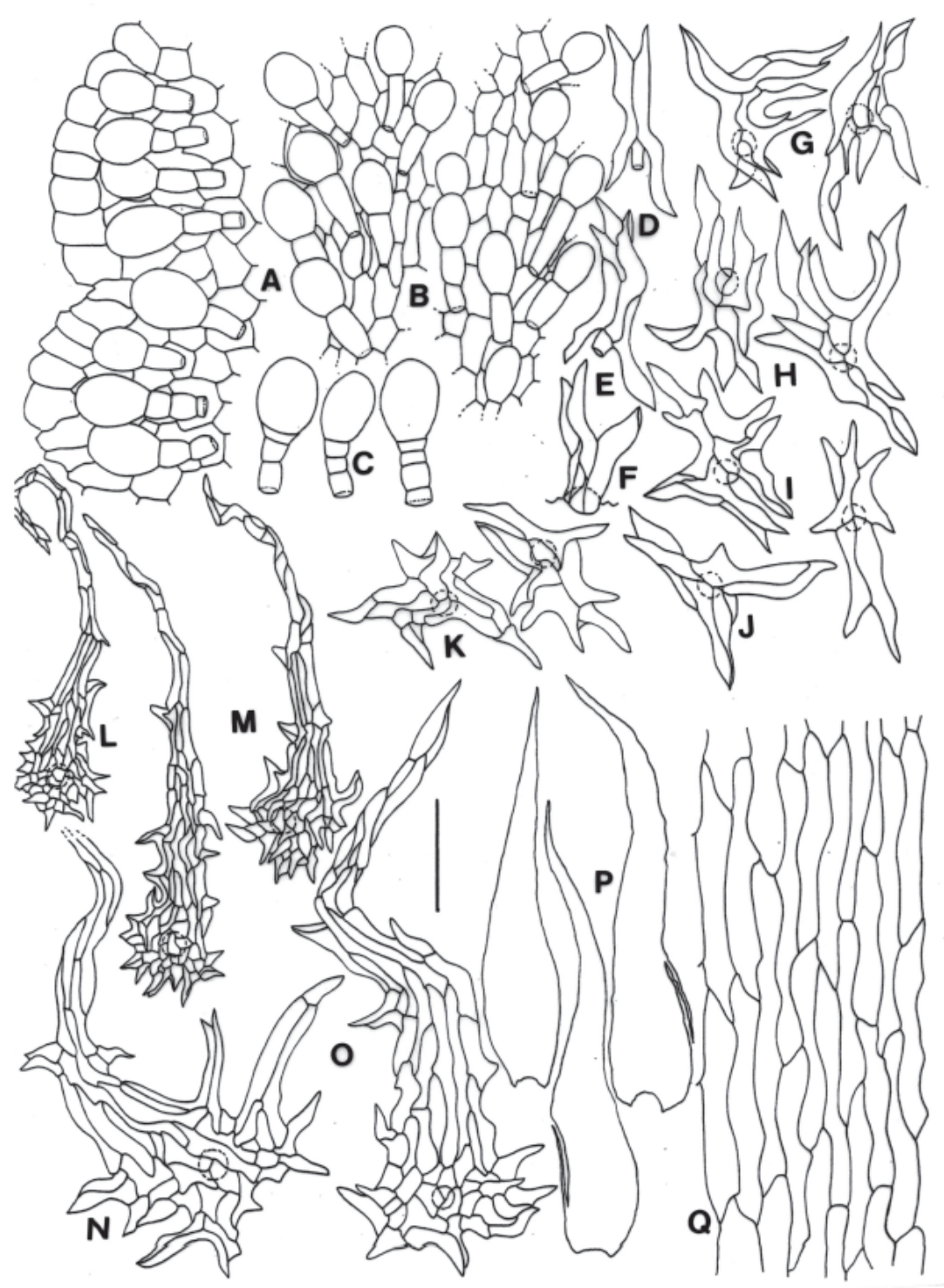

Figura 2. Indumento de B. cordatum. A-C: Pelos glandulares mucilaginíferos. A: Pelos superficiales de la epidermis del circino. B: Pelos sobre venas juveniles del circino. C: Pelos superficiales de lámina adulta. D-F: Escamas superficiales peltadas de la epidermis del circino. G-J: Escamas peltadas de la epidermis, venas y costas de la fronda tenueral. K: Dos escamas de la lámina adulta. L-O: Escamas de las venas, costas, raquis y estípite adultos. P: Tres escamas rizomáticas y de la base del estípite. Q: Detalle de la escama ilustrada en P. Barra= 100 um en A, C; 80 um en B; 0,5 mm en D-J; $1 \mathrm{~mm}$ en K; $2 \mathrm{~mm}$ en L-M; 2,5 $\mathrm{mm}$ en N-O; $1 \mathrm{~cm}$ en P; $200 \mu \mathrm{m}$ en Q. Indument of B. cordatum. A-C: Grandular mucilaginiferous hairs. A: Superficial hairs on the epidermis of the croziers. B: Hairs on the juvenile veins of the crozier. C: Superficial hairs of the adult lamina. D-F: Superficial, peltate scales of the epidermis of the crozier. G-J: Peltate scales of the epidermis, costa, and veins of a teneral frond. K: Two scales of the adult lamina. $L$ $O$ : Scales of adult veins, costa, rachis and stipe. P: Three escales from rhizoma and base of stipe. $Q$ : Detail of the scales illustraded in P. Bar $=100$ um in $A, C ; 80$ um in B; $0.5 \mathrm{~mm}$ in D-J; $1 \mathrm{~mm}$ in K:2 $\mathrm{mm}$ in $L-M ; 2,5 \mathrm{~mm}$ in $\mathrm{N}-\mathrm{O} ; 1 \mathrm{~cm}$ in $P$; $200 \mu \mathrm{m}$ in $Q$. 
Escamas más pequeñas, similares, se encuentran sobre la superficie de la lámina (fig. $2 \mathrm{~K}$ ). Los pelos son superficiales, esparcidos, similares a los descritos para los circinos (fig. 2 C).

Los modelos epidérmicos son sinuosos en epifilos e hipofilos y la epidermis está formada por células con las paredes uniformemente engrosadas. La ontogenia de la epidermis indica que el modelo inicial es poligonal en los circinos y frondas en despliegue y que las plantas de menos de 40 $\mathrm{cm}$ de altura tienen células epidérmicas que aún no han desarrollado sus sinuosidades características (fig. 3 A-B), presentando un modelo subsinuoso, con paredes epidérmicas más delgadas que en los ejemplares más grandes. Los epifilos adultos difieren en modelo de los hipofilos: en los primeros las células son grandes y hasta 3-4 veces más largas que anchas, con ondulaciones profundas y desiguales (fig. 3 C, E, G), mientras que en los hipofilos adultos las células son de menor tamaño y sus ondulaciones compuestas y más frecuentes (Fig 3 D, F, H). En sección transversal la epidermis es moderadamente papilosa y en algunos ejemplares que crecen a mayor latitud o a mayor altura sobre el nivel del mar la epidermis puede presentar estrías finas.

Los estomas son elípticos, algo hundidos, especialmente en ejemplares expuestos o de altura. Predominan los tipos diacíticos y anomocíticos (Prabhakar, 2003), las dimensiones encontradas son (47) 57 (62) x (28) 30 (36) um y la densidad estomática es de (8) 12 (28) estomas / $\mathrm{mm}^{2}$. Los modelos epidérmicos, como los tamaños y densidades estomáticos son notablemente coincidentes en materiales de distintas localidades (fig. 3 D, F, H).

Los indusios son membranáceos en plantas jóvenes, firmes y rígidos en las adultas. Tienen margen subentero a eroso o

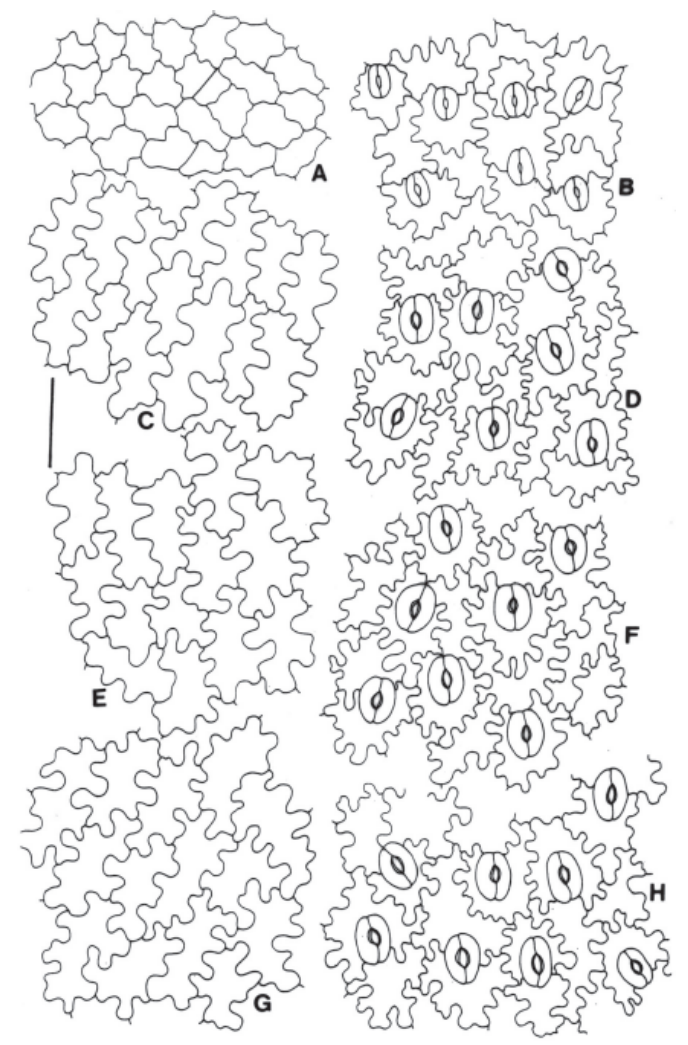

Figura 3. Modelos epidérmicos en B. cordatum. A-B: Epidermis en planta juvenil de Argentina, Río Negro, Prada s/n (MA). C-D: Epidermis adulta en ejemplar de Brasil, Minas Gerais, Smith 2172 (BA). E-F: Epidermis adulta, en ejemplar de Colombia, Chocó-Antioquía, Lellinger \& de la Sota 855 (LP). G-H: Epidermis adulta en ejemplar de Australia, Botany Bay, Neés. n. (MA 213373). Izquierda, epifilos; derecha, hipofilos. Barra $=100 \mu \mathrm{m}$. Epidermis of the pinnae of $B$. cordatum. A-B: Epidermis in a juvenile plant from Argentina, Río Negro, Prada s/n MA). C$D$ : Adult epidermis in a specimen from Brasil, Minas Gerais, Smith 2172 (BA). E-F: Adult epidermis in a specimen from Colombia, ChocóAntioquía, Lellinger \& de la Sota 855 (LP). G$H$ : Adult epidermis in a specimen from Australia, Botany Bay, Neé s. n. (MA 213373). Left, epiphylls; right, hypophylls. Bar= $100 \mu \mathrm{m}$. 
lacerado, discontinuamente papiloso en los márgenes.

Las esporas son monoletas, elipsoidales, con perisporio crestadoreticulado. La cara proximal es levemente convexa en vista ecuatorial y la lesura puede ser o no sobresaliente. El perisporio es externamente crestado-reticulado, con crestas sobresalientes y con las áreas entre crestas cubiertas con micro-ornamentación filamentosa (fig. 4 A-D), con procesos filiformes entremezclados y parcialmente fusionados formando una especie de red que se dispone sobre una superficie lisa a finamente rugulada (fig. $4 \mathrm{~F}$ ). El perisporio tiene una capa externa compacta, que es la crestado-reticulada descrita y una capa fibroso-fenestrada por debajo, con procesos unidos formando una red densa (fig. 4 D-E). Una capa más interna, irregularmente granular, con gránulos gruesos, parece quedar adherida al exosporio (fig. 5 A-C). La lesura es relativamente larga y puede alcanzar 57-60 $\mu \mathrm{m}$, ocupando casi toda la cara proximal de la espora. Es poco sobresaliente, puede ser casi recta, sinuosa y ondulada o bien con un margen en forma de labio cuya prominencia puede variar en esporas de distintas localidades.

\section{Tratamiento taxonómico}

Blechnum cordatum (Desv.) Hieron., Hedwigia 47: 239 (1908).

Lomaria cordata Desv., Mag. Neuesten Entdeck. Gesammten Naturk. Ges. Naturf. Freunde Berlin 5: 330 (1811) [basión.]

Tipo: [Chile]: "Perou- Concepcion", Dombey (Holótipo P-JU 1368!)

Lomaria chilensis Kaulf., Enum. filic. 154 (1824). Blechnum chilense (Kaulf.) Mett., Fil. lechl. 1: 14 (1856). Ind. loc.: Habitat in Chile, Chamisso.

Lomaria ornifolia C. Presl, Reliq. haenk. 1: 51 (1825). Blechnum ornifolium
(C. Presl) Ettingsh., Denkschr. Kaiserl. Akad. Wiss. Wien. Math. Naturwiss. K1. 23: 61 (1864). Ind. loc. : Hab. in Peruvia [Haenke]. Citado en la sinonimia de $B$. cordatum por Hieronymus, Hedwigia 47: 239 (1908).

Lomaria serrulosa Desv., Mém. Soc. Linn. Paris 6: 290 (1827). Tipo: Crescit in Peruvia (Holótipo P).

Lomaria gilliesii Hook. \& Grev., Icon. filic. 2, t. 207 (1831). Orthogramma gilliesi (Hook. \& Grev.) C. Presl, Abh. Königl. Böhm. Ges. Wiss., 6: 481 (1851). Blechnum gilliesii (Hook. \& Grev.) Mett. Fil. hort. bot. Lips. 64 (1856). Tipo: Chile, Gillies (Holótipo K!, foto US, C. V. Morton negativo 11355).

Lomaria arborescens Klotzsch \& H. Karst., Linnaea 20: 347 (1844). Blechnum arborescens (Klotzsch \& H. Karst.) Hieron., Hedwigia 47: 239 (1908). Ind. loc.: Venezuela: In montibus Caracas, Moritz 116. Síntipo: Venezuela: Mérida, Moritz n. 299. Galipán, Moritz 300. Colombia: Karsten n. 58 (Coll. I). Karsten 25 (Coll. II) [Síntipo B-Willd!] .

Lomaria regnelliana Kunze, Linnaea 22: 576 (1849). Blechnum regnellianum (Kunze) C. Chr., Index filic., Suppl. 1: 17 (1913). Ind. loc.: "Brasilia, prov. Minas Gerais, fecha 18/843? (fecha confusa), Regnell 1490". Tipo: Brasil: Minas Gerais, Caldas, Regnell 1490 (Holótipo B-Willd.!, fragm. HB, isótipos BM, US!).

Lomaria reedii Phil. Anales Univ. Chile 43: 581 (1873). Blechnum reeddi (Phil.) Espinosa, Revista Chilena Hist. Nat. 41: 337 (1938). Blechnum chilense (Kaulf.) Mett. var. reeddi (Phil.) Looser, Revista Univ. (Santiago) 32 (2): 47 (1947). Ind. loc.: De la isla de Juan Fernández; de las regiones más australes del continente la trajo, igualmente, el Comandante Simpson (Holótipo SGO).

Blechnum itatiaiense Brade, Arq. Inst. Biol. Veg. 1(3): 225 (1935). Tipo: Brasil: 
Serra do Itatiaia, $2000 \mathrm{~m}$, Brade 10115. Lectótipo R, designado por Oliveira Dittrich (2005).

Blechnum macahense Brade, Anais Primeira Reunião Sul-Amer. Bot. 2: 6 (1938). Tipo: Brasil: Rio de Janeiro, Macaé, Frade de Macaé, ca. 1200 m, 19.VI (1937), Brade15801 \& Santos Lima (Holótipo RB, isótipo HB).

Blechnum raddianum Rosenst., Hedwigia 46: 91 (1907), nom. nov. para Lomaria brasiliensis Raddi, Opusc. Sci. 1: 50 (1825), nom. illeg., non Blechnum brasiliense Desv. (1811). Struthiopteris brasiliensis (Raddi) Maxon \& C. V. Morton, Bull. Torrey Bot. Club 66: 43. (1939). Ind. loc.: Invenitur saepissima ad margines nemorum humidorum in vallibus prope Tijuccam. Haec species media est inter Lomariam lineatam et striatam, cum quibis convenit magna ex parta suis specificis characteribus. Tipo: Brasil: Rio de Janeiro: Tijuca, Raddi. (isótipo FI).

Blechnum simile Sehnem, in P. Reitz, F1. Ilustr. Catarin. (BLEC): 56 (1968). Tipo: Brasil : Rio Grande do Sul, Aparados da Serra, Passo do Guarda, 800 m, Sehnem 5861 (Parátipo PACA).

Blechnum peruvianum Hieron., Hedwigia 47: 245 (1908). Tipo: Perú: Moyobamba, Rio Huallaga, Stubel 1105 (Holótipo B-Willd.B 200032825 B 20 0032825!).

Blechnum chilense (Kaulf.) Mett. f. imbricatum G. Kunkel, Nova Hedwigia 13 (3-4): 337. (1967). Ind. loc.: [Chile] [Isla] Mocha, Quebrada de Las Casas, $80 \mathrm{~m}$, Quebrada Ortigas, Oktober 1958, G. Kunkel 6390 (PTER Siehe Anm, S. 339).

Plantas terrestres, calatifrondas de hasta $250 \mathrm{~cm}$ de altura. Rizoma erecto, corto, grueso, de hasta $50-60 \times 15-16 \mathrm{~cm}$, no estolonífero, escamoso. Escamas rizomáticas lanceoladas, largamente atenuadas, de hasta
$3 \times 0,5 \mathrm{~cm}$, concoloras, pardo-amarillentas a castañas, planas, a veces con laceraciones piliformes en el ápice y los márgenes. Frondas dimórficas, las estériles arqueadas, de $35-200 \mathrm{~cm}$, las fértiles erguidas, de 30 $210 \mathrm{~cm}$. Estípite 1/4-1/2 del largo total de la fronda, verde y flexible en plantas jóvenes, leñoso, rígido, acanalado en plantas adultas, internamente masivo, dictiostélico, con escamas basales similares a las del rizoma, castañas, translúcidas. Lámina estéril de lanceolada a lanceolado-elíptica, imparipinnada; pinna terminal lanceolada conforme con las laterales, siempre más larga que las adyacentes y con el ápice agudo; pinnas laterales de 5-20 x 1-3 cm, de lanceoladas a lanceolado-oblongas, con ápice agudo, base cordada simétrica o algo asimétrica, brevemente pecioluladas, coriáceas, estriadas o rugosas en plantas de mayores altitudes o latitudes, con margen aserrado y finamente dentado. Aeróforos de contorno y prominencia variables, a veces poco visibles o bien ausentes. Indumento en raquis, costas e hipofilos, formado por pelos y escamas, éstas lanceoladas anchas, con procesos laterales agudos, de hasta $0,5-0,6$ x 0,15-0,20 cm, mezcladas con pelos glandulares capitados esparcidos o densos, sobre ejes y lámina; circinos escamosos y con abundantes pelos glandulares con 3-4 células, capitados, secretores de mucílago. Venación simple, venas geminadas y bifurcadas a distancias variables de la costa. Pinnas fértiles casi tan largas como las estériles y más numerosas, con lámina extremadamente reducida, margen incurvado, subdenticulado y cenosoros cubriendo casi toda la superficie; indusio de hasta $0,4 \mathrm{~cm}$ de ancho, de subentero a eroso o lacerado, membranáceo, firme, parcialmente papiloso en los márgenes, continuo. Esporas monoletas, castañoamarillentas, elipsoidales, de (60) 62 (74) x (44) 47 (57) $\mu \mathrm{m}$, con perisporio reticulado- 


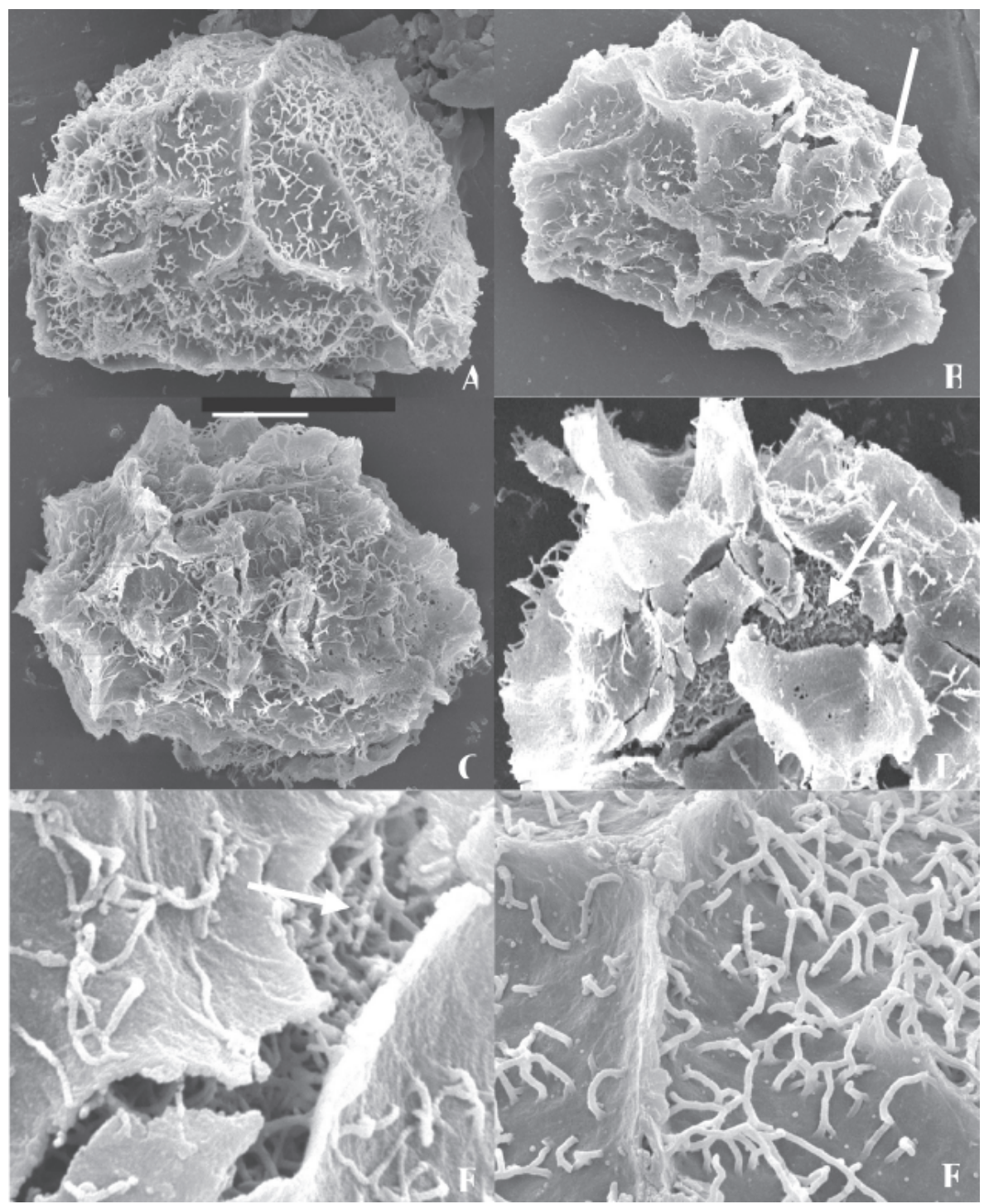

Figura 4. Perisporio de las esporas de B. cordatum. A-D: Esporas con perisporio crestado-reticulado y micrornamentación filamentosa. A: En material de Colombia, Chocó-Antioquia, Lellinger \& de la Sota 855 (LP). B: En material de Chile, Concepción, Pérez Moreau 22912 (BA). C-D: En material de Australia, Botany Bay, Neé s. $n$. (MA 213373). E: Detalle del perisporio en B (área señalada por flecha), capa externa compacta, crestado-reticulada, con micrornamentación filamentosa y capa interna fibroso-fenestrada. F: Detalles de micrornamentación filamentosa en las áreas entre crestas, en A. Barra $=10 \mu \mathrm{m}$ en A-D, $2 \mu \mathrm{m}$ en E-F. The perispore of spores of B. cordatum. A-D: Cristate-reticulate perispore, with filamentose micrornamentation. A: Specimen from Colombia, Chocó-Antioquia, Lellinger \& de la Sota 855 (LP). B: Specimen from Chile, Concepción, Pérez Moreau 22912 (BA). CD: Specimen from Australia, Botany Bay, Neé s. $n$. (MA 213373). E: Detail of perispore in B (area pointed by arrow), external compact, cristate-reticulate layer, with filamentose micrornamentation, and internal fibrose-fenestrate layer. F: Details of filaments in A. Bar= 10 um in A-D, 2 um in E-F. 
crestado de 6,2-7,3 um de espesor y exosporio de 2,5-3,3 $\mu \mathrm{m}$; crestas del perisporio sobresalientes y áreas entre crestas con filamentos. Gametófitos desnudos. $2 n=66$.

Distribución geográfica y ecología. Blechnum cordatum es una especie pantropical; se encuentra en los Dominios Caribe, Amazónico, Chaqueño y Andinopatagónico de la Región Neotropical y en el Dominio Subantártico de la Región Antártica (Cabrera \& Willink, 1973), es decir en Mesoamérica, Antillas, Sudamérica (Colombia, Venezuela, Ecuador, Perú, Bolivia, Brasil, Paraguay, Argentina, Chile y Uruguay), archipiélagos de Juan Fernández, Hawái y SE de Australia. Se han estudiado dos pliegos de la Expedición Malaspina: en uno de ellos se indica una localidad de Australia oriental, "Ex Botany Bay, in Nova Hollandiae" y en el otro se indica "Ex Nova Hollandia y Marianas" con ejemplares cuyos caracteres anatómicos y esporales permiten atribuir las plantas a $B$. cordatum. Si bien la forma de expresar la localidad en el segundo pliego es confusa, en ambos casos coincide en la mención de Australia oriental (Nueva Gales del Sur), lo que extiende la presencia de la especie hasta Oceanía. Crece en ambientes de selva pluvial, nublada y monzónica, en bosques tropófilos y perennifolios, a lo largo de caminos y en áreas boscosas húmedas, hasta pantanosas, hendiduras o grietas de rocas y en lechos de inundación; es relativamente abundante en las selvas húmedas de las vertientes orientales andinas, en selvas montanas y pedemontanas y en los páramos, hasta los $3500 \mathrm{~m}$ de altitud. En Brasil y Paraguay puede ser umbrófila en selvas semideciduas o en áreas ribereñas pero no es estrictamente selvática y también crece en áreas abiertas y en los campos, casi desde el nivel del mar hasta los $2000 \mathrm{~m}$. Los ejemplares de Juan Fernández tienen caracteres similares al material americano de áreas selváticas, mientras que los australianos son más rígidos y se parecen más al material austral de la especie o a las formas que crecen a mayor altitud.

\section{Material estudiado}

ARGENTINA: BUENOS AIRES: Cerro Curamalal Grande, Scala 3010 (LP). Buenos Aires: 950 m, de la Sota 6500 (LP). Sierra de la Ventana, Harrington 3 (SI 55734). Sierra de La Ventana, Cabrera 4455 (LP). Sierra de La Ventana, Alboff 217 (LP). Tornquist, Sierra de la Ventana, Cabrera \& Fabris 51 (LP). Bajada de La Ventana, de la Sota 6500 (LP). Sierra de La Ventana, Valle de las Vertientes, Spegazzini 17879 (LP). Sierra de La Ventana, Arroyo San Bernardo, Proyecto Ventania 717 (LP). Sierra de La Ventana, Capurro 1112 (BA). Sierra de La Ventana, Lorentz 728 (MVM*). Saavedra, Cerro Curamalal Grande, 700 m, Scala 3010 (LP). Balcarce, Sierra Volcán, Pa. del Abra, Martínez Crovetto 1539 (LP). Balcarce, Sierra Vigilancia, Gallardo 52166 (BA). Saavedra, Cerro Curamalal Grande, Villamil 5098 (LP). Pigüé, Burkart 4586 (SI). CÓRDOBA: Sierra de Achala, Los Gigantes, Kurtz 6929 (LP, SI). [Córdoba] Hieronymus 203 \& Hieronymus 330 (CORD) y Kurtz 6929 (LP). Sierras Grandes, Gautier 1 (LP). CHUBUT: Parque Nacional Los Alerces, sporde orientali del lago Futalufquen, presso la Ostaria Cume Hué, Pichi-Sermolli \& Bizarri 7383 (LP). Futaleufú, Parque Nacional Los Alerces, Lago Cisnes, Roig \& al. s. n. (LP). NEUQUÉN: Minas-Lagunas, Epulauquén, Bosque Encantado, Correa \& Fortunato 9896 (LP). Epulauquén, Bosque Encantado, Gentili \& Pérez 1 (LP). Bosque Encantado, Boelcke \& Correa 7032 (LP). San Martín de los Andes, cascada de Quila-Quina, 500-650 m, 28-11-2005, Prada s/n (LP, MA). San Martín de los Andes, Gentili \& Pérez 1 (LP). San Martín de los Andes, Bridarolli 2043 (LP). RÍO NEGRO: Bariloche, Nahuel Huapi, Puerto Pañuelo, Burkart 5565 (SI). Bariloche: Lago Nahuel Huapi, Brazo Blest, camino a la Cascada de los Cántaros, 3-12-2005, Prada s/n (LP, MA). San Carlos de Bariloche, camino a Bahía López, 


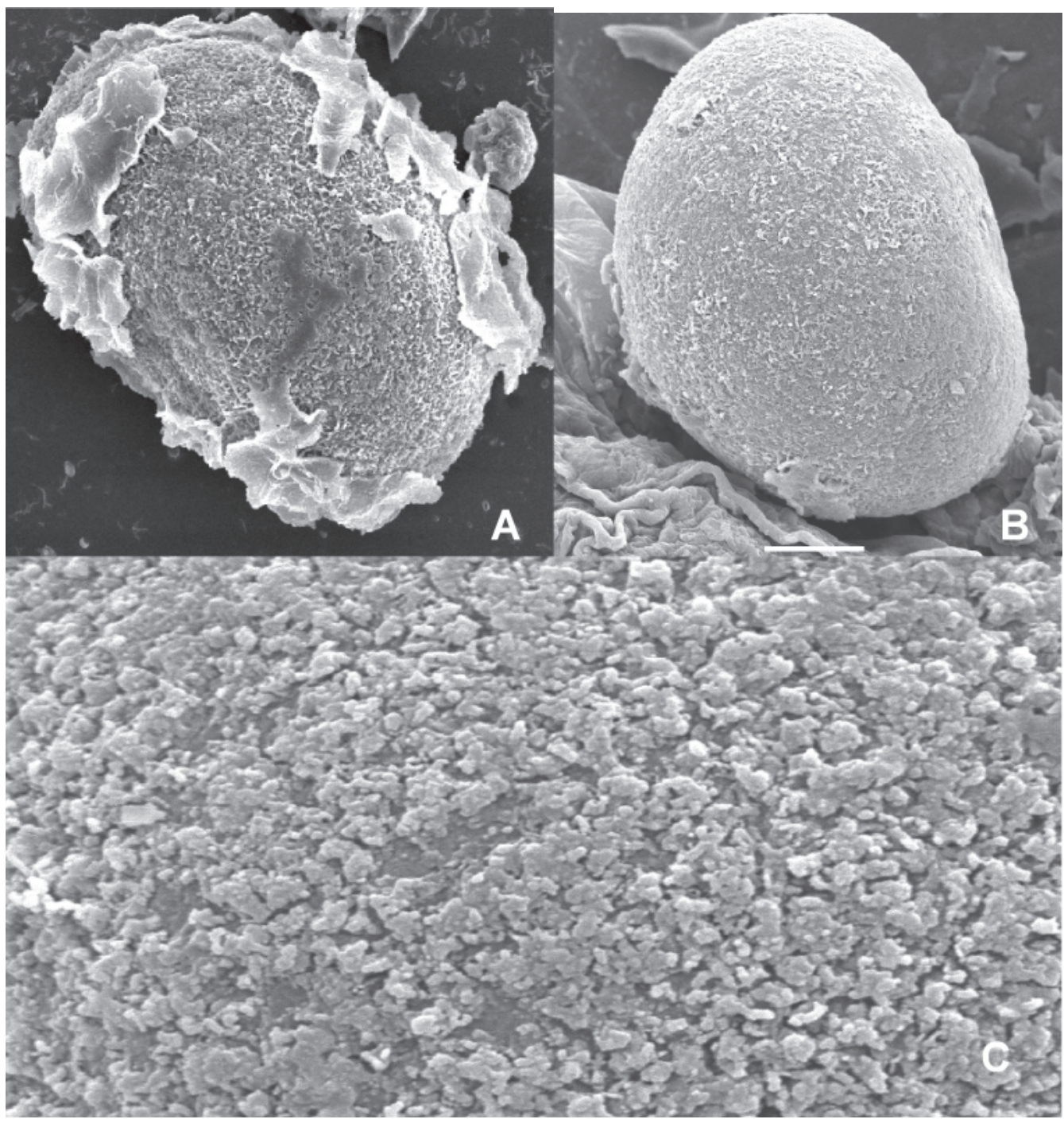

Figura 5. Capas del perisporio de las esporas de B. cordatum. A: Espora con la capa externa del perisporio parcialmente desprendida, en material de Australia, Botany Bay, Neé s. n. (MA 213373). B: Espora con la capa interna granular del perisporio, que queda adherida al exosporio, en material de Chile, Juan Fernández, Isla Robinson Crusoe, Meyer 9453 (LP). C: Detalle de la capa granular ilustrada en B. Barra $=10 \mu \mathrm{m}$ en A-B, $2 \mu \mathrm{m}$ en C. Perispore layers in spores of B. cordatum. A: Spore with the external shedded perispore layer, in a specimen from Australia, Botany Bay, Neé s. $n$. (MA 213373). B: Spore with inner granular layer of perispore, which remains adhered to the exospore, in a specimen from Chile, Juan Fernández, Isla Robinson Crusoe, Meyer 9453 (LP). C: Detail of the granular layer illustrated in B. Bar= $10 \mu \mathrm{m}$ in $A-B, 2 \mu \mathrm{m}$ in $C$. 
Burkart 12047 (BA). Sin loc, "Río Negro", Corte 33 (LP). Parque Nacional Nahuel Huapi, Puerto Blest, Delucchi 1554 (LP). Parque Nacional Nahuel Huapi, Puerto Blest, Cabrera \& Job 252 (LP). Parque Nacional Nahuel Huapi, Lago Espejo, Cabrera 5975 (LP). Parque Nacional Nahuel Huapi, Lago Gutiérrez, Birabén \& Birabén 629 (LP). Parque Nacional Nahuel Huapi, entre Puerto Blest \& Laguna Frías, Núñez s. n. (BA 56524). Laguna Frías, Corte 32 (LP). Camino de Puerto Alegre a Puerto Blest, ca. Lago Frías, prope Bariloche, Prada $s / n$ (LP, MA).SALTA: $1750 \mathrm{~m}$, Cassá de Pazos \& al. $287 a$ (LP). Santa Victoria, camino Los Toldos a Lipeo, Palaci 514 (LP, MCNS). TIERRA DEL FUEGO: Península Mitre, Bahía Buen Suceso, Materi 74264 (BA). Sin loc., Moore 858 (LP).

AUSTRALIA: [SYDNEY] Ex Botany Bay in Nova Hollandiae, Née s/n (MA 213373). Ex Nova Hollandia y Marianas, Née s/n., Exp. Malaspina (MA 230579). NUEVA GALES DEL SUR: Blue Mountain, 04-1897, Camfield s.n. (MVM*).

BOLIVIA: LA PAZ: Nor Yungas, Milluguaya, Buchtien 5016 (NY 813957). Sud Yungas, $9 \mathrm{~km}$ de Huancané en la carretera hacia San Isidro, Smith \& Smith 13102 (LP, LPB*, MO*). Saylapata-Ayopaya, Cárdenas 3151 (LIL). SANTA CRUZ: Florida, $7 \mathrm{~km}$ (by road) NE of crossing of Río Paredones north of Achiras Camping resort, on trail to Abra Los Toros, $M$. Nee $49703\left(\mathrm{NY}^{*}\right)$. Florida, near bottom of valley of Quebrada Guapurucillo, between mountains of massive red sandstone of Serranía de la Cruz to the NE and Cerro Guapurucillo to the south, along dirt road to Bella Vista, $2 \mathrm{~km}$ SE from turnoff (the turnoff ca. $3 \mathrm{~km}$ E of Cuevas from highway from Santa Cruz to Samaipata), Nee 48370 $\left(\mathrm{NY}^{*}\right)$.

BRASIL: MINAS GERAIS: Lindberg 614 (NY*). Serra do Espinaço, E of Diamantina, Irwin \& al. 28023 (NY*). Serra do Espinaço, ca. 11 $\mathrm{km}$ of Gouveia, Municipio de Datas, km 286 on MG 289, 1300 m, Anderson \& al. 35577 (NY*). Valley of the Rio Campo Bello, vicinity Monte Serrat, Mt. Itatiaya, Estacão Biologica, Smith 2172 (BA). Ouro Preto, de la Sota 2645 (LIL). Municipio Mariana, Pasagem de Mariana, Labouriou 1030 (LIL). PARANÁ: Curitiba, Rod. do Café, rio Capivari (Mun. Tibagi), Hatsbach
38077 (SI). Porto Amazonas, Fazenda San Luis, Hatschbach 10793 (LIL). RIO DE JANEIRO: Nova Friburgo, margen del rancho La Sagrada Familia, Capell S. J. s/n. (MA 186634). Porto Alegre, San Francisco de Paula, Villa Oliva, Rambo 31244 (LIL). RIO GRANDE DO SUL: Santa Cruz, Jürgens \& Ittier 69 (BA, SI). SANTA CATARINA: Joinville, A. Schmalz 82 (NY*). Joinville, Schmalz 159 (MVM*). Km 26 da estrada Itaipava-Teresópolis, 1200 m, Pabst 7153 (LP). Municipio Põrto União, alt. ca. $750 \mathrm{~m}$, Smith \& Reitz 8830 (SI). Lages, 950 m, Sehnem 68898 (BA). Chapecó, Fazenda Campo São Vicente, $24 \mathrm{~km}$ W of Campo Eré, 900-100 m, Smith \& al. 9370 (SI). SÃO PAULO: São Jose do Barreiro, Hoehne 17679 with A. Gehrt (NY*). En el camino de Curitiba a São Paulo, lugar llamado Santo Domingo, Roig 3978 (LIL ).

CHILE: CONCEPCIÓN: Talcahuano, Rocoto, de la Sota 6020 (LP). Departamento Tranco, Colcura, Pérez Moreau 22912 (BA). VALDIVIA: Todos los Santos, Haumann 20 (BA). LLANQUIHUE: Alerce, Cabrera 3697 (BA). ISLAS DE JUAN FERNÁNDEZ: Isla Robinson Crusoe (ex Mas a Tierra), Quebrada de La Mata Maqui, ca. $900 \mathrm{~m}$, enroute to Correspondence Camp, Meyer 9453 (LP). On path from Pueblo San Juan Bautista to Mirador Serlkirk, Stuessey \& Sanders 5016 (MA). REGIÓN VII: Linares, a lo largo del río Achibueno, desde junta estero Las Ánimas a junta estero Riecillo, Ruiz \& López 1217 (MA). REGIÓN X: Aguas Calientes, Parque Nacional Puyehue, Debandi 1 (LP). Ofqui? San Rafael, Hicken 3 (SI). Los Lagos, Cabrera 3697 (LP). Palena, $22 \mathrm{~km} \mathrm{~S}$ of Chaltén on road to Puerto Cárdenas, Stuessey \& al. 7087 (MA).

COLOMBIA: ANTIOQUIA: en potreros cercanos a La Ceja, Barkley \& al. 1531 (LIL). BOYACÁ: entre Arcabuco y Villa de Leyva, camino a Las Coloradas, $2900 \mathrm{~m}$, Jaramillo Mejía, Murillo \& van der Hammen 3049 (MA). Camino del Alto de Onzaga a Soatá, en el Valle Lateral, Jaramillo Mejía \& van der Hammen 4177 (MA). CUNDINAMARCA: Carretera de La Mosquera a La Mesa, parte baja del Robledal de La Merced, 2700 m, Jaramillo Mejía, Murillo \& van der Hammen 4704 (MA). CHOCÓANTIOQUÍA: At and on both sides of the principal ridge $\mathrm{W}$ of La Mansa, at ca. Km 105.5 
of the ciudad Bolivar, Quibdo Road, Lellinger \& de la Sota 855 (LP, US*). CHOCÓ: W of El Siete along the Ciudad Bolívar-Quibdo Road, $1500 \mathrm{~m}$, Lellinger \& de la Sota 872 (LP, US*).

COSTA RICA: ALAJUELA: Vicinity: Evans \& Bowers 2932 (MO*). CARTAGO: Tapantí, ca. $15 \mathrm{~km} \mathrm{~S}$ of Paraíso, trail through wet forest, Mickel 2318 (LP, US*). SAN JOSÉ: vicinity of La Hondura, $4 \mathrm{~km} \mathrm{~S}$ to $1 \mathrm{~km} \mathrm{~N}$ on route 22, between Volcán Irazu and Volcán Barba, 1200-1500 m, Mickel 2225 (LP, US*). Vicinity of San José, Greenman \& Greenman 5383 (MO*).

DOMINICA: St. Paul Parish, frequent along Banks of the rivière Deux Branches where it crosses the road $0.8 \mathrm{mi}$ of Port Cassé, Lellinger $\&$ de la Sota 391 (LP, US*). Saint David Parish, trail between Boeri lake and Freshwater Lake above Laudat, Hill 29004 (UC*).

ECUADOR: TUNGURAHUA, crescit in sylvas suband. occ. volc. Tungurahua, Sodiro $s$. n. (BA 57779). BOLÍVAR: Road above Balzapamba, Haught 3300 (LIL).

HAWÁI: OAHU: Manoa valley, Lyon Arboretum, Wilson s. n. (BISH 622596 \& 622597*).

JAMAICA: PORTLAND: Trail between Portland Gap and Blue Mountain, Papenfuss s. n. (UC*).

MEXICO: CHIAPAS, Cerro Huaitepec, 5 $\mathrm{km}$ NO of Ciudad de Las Casas (San Cristóbal), Little Jr. \& Sharp 9928 (UC). OAXACA: Ixtlán de Juárez, Krueger \& Gillespie 37 (UC).

PARAGUAY: CAAGUAZÚ: In viciniis Caaguazú, Hassler 9389 (NY*). Caaguazú, Sparre \& Vervoorst 1733 (LIL). Barranca del río Verde, Cabezadita, Yerbales, Rojas 3803 (MVM*, PY*). AMAMBAY: San Luis, Schwarz 12260 (LIL). PARAGUARÍ: Salto Piraretá, Sparre \& Vervoorst 347 (LIL).

PERÚ: CAJAMARCA, La Chota, Sagástegui 12893 (F*). CUZCO: sin loc., Gentry $23449\left(\mathrm{~F}^{*}\right)$. La Convención, Cordillera Vicabamba, $5 \mathrm{~km}$ N of Aguas Calientes, Solomon 3169 (USM). JUNÍN/CUZCO: León s. n. (F 224860*). PASCO: Oxapampa, León \& Young 1730 (USM). Quillasú, Soukup 3284 (LIL). PIURA: Ayabaca, bosque de Huamba, Cano 1612 (USM). SAN MARTÍN: Provincia Mariscal Cáceres, Rio Abiseo National Park, Young 1768 (USM).
VENEZUELA: TRUJILlO: Dorr \& al. 8309 (US*). Trujillo, Stergios \& Zambrano 16684 \& 17683 (US*). Boconó, Parque Nacional Guaramacal, vertiente norte, camino Laguna de los Cedros, cresta del páramo, Stergios \& Palacios 18648 \& 18648 (US*).

\section{DISCUSIÓN}

Blechnum cordatum es una especie con un área de distribución amplia, pantropical; es considerada abundante y poco vulnerable. Aunque aparece descrita en numerosos trabajos florísticos, las descripciones son incompletas y no existe un análisis de morfología comparada en especímenes de toda su área de distribución, como el que aquí se ha efectuado.

De acuerdo con lo que se ha revisado aquí, hay rasgos distintivos, que permiten caracterizarla, así como otros que presentan cierto grado de variación, a veces debida a las distintas fases del crecimiento de las plantas.

Son rasgos diagnósticos los tipos de escamas rizomáticas y peciolares, las frondas marcadamente dimórficas, los estípites con tres meristelas en plántulas y 14-16 en ejemplares adultos, la arquitectura de la fronda, los tipos y distribución del indumento en ejes y lámina, la presencia, contorno y tamaño de la pinna terminal en relación con las laterales, los márgenes, el patrón de venación, los modelos epidérmicos y tipos estomáticos de las pinnas laterales y la ornamentación de las esporas. Los tamaños estomáticos y esporales de $B$. cordatum varían escasamente en materiales de distintas localidades del área y no hay evidencias morfológicas, por el momento, de ploidías intraespecíficas diferentes. El único dato citológico relacionado con esta especie es el recuento efectuado por Jara-Seguel et al. (2006) como B. chilense, en el que se registra un número diploide de $2 \mathrm{n}=66$. Las esporas 
son muy características y su morfología es constante en ejemplares de toda el área, algo que no se conocía previamente y que tiene interés en la sistemática del género. La presencia y desarrollo de los aeróforos, la textura de la lámina, la profundidad del aserrado marginal y la lesura labiada en las esporas son algo inconstantes.

La dictiostela de los estípites adultos de B. cordatum, con hasta 16 meristelas, deviene de la fragmentación de una estela juvenil con 3 haces vasculares, un número también registrado para todos los taxones del grupo B. penna-marina (Rolleri \& Prada, $2006 \mathrm{~b}$ ) y todos los taxones relacionados con B. occidentale. Esta coincidencia en el número de haces de la estela juvenil de especies con esporófitos arborescentes y de la estela adulta de especies con esporófitos herbáceos, sugiere que el hábito arborescente podría ser un carácter derivado en el género.

Los gametófitos de $B$. cordatum fueron estudiados en material austral de la especie por Pérez-García et al. (1996), quienes además, ilustraron epidermis juveniles con tricomas y células epidérmicas con sinuosidades someras, similares los observados aquí en plántulas.

De las especies del grupo $B$. lineatum (Tryon \& Tryon, 1982) que se han podido revisar hasta el momento, Blechnum proliferum, que crece en Mesoamérica (Costa Rica y Panamá) y Sudamérica (desde Venezuela y Colombia hasta Brasil) parece ser la más afín con $B$. cordatum, de la que se distingue por la presencia de yemas en las porciones apicales de las frondas, las pinnas lanceoladas con bases redondeadas, acroscópicamente cuneadas, el patrón de venación, los modelos epidérmicos y las esporas crestadas sin micro-ornamentación filamentosa.

Las especies del grupo $B$. lineatum (Tryon \& Tryon, 1982) o grupo B. cordatum (Oliveira Dittrich, 2005), requieren ser mejor estudiadas para establecer sus límites y analizar su afinidad con B. cordatum. Moran (1995) trató a B. schiedeanum 'para Mesoamérica, incluyó $B$. costaricense entre sus sinónimos y la consideró muy similar a $B$. cordatum. Ese autor también trató como especie distinta a $B$. falciforme. Mickel \& Smith (2004) señalaron las semejanzas entre B. falciforme, B. schiedeanum, B. varians y $B$. cordatum, aunque las mantuvieron como taxones diferentes. El material estudiado en el presente trabajo incluyó ejemplares determinados como B. christii C. Chr., B. falciforme, B. lineatum, B. schiedeanum y $B$.varians, que tenían los caracteres que aquí se han considerado específicos de $B$. cordatum y como tales se han incluido en el material atribuido a esta especie.

Blechnum ryannii (Kaulf.) Hieron., de Santa Lucía y Dominica y B. striatum $(\mathrm{Sw})$. C. Chr. (nom. illeg.), han sido considerados coespecíficos por Steyermark et al. (1995) y estrechamente relacionados con $B$. cordatum. El material visto aquí, determinado como $B$. striatum pertenece a $B$. cordatum o a B. proliferum. El material de Costa Rica determinado como B. striatum vel aff. (Greenman \& Greenman $5383 \mathrm{MO}$ ) y el ejemplar determinado como B. ryanii, de Dominica (Lellinger \& de la Sota 391 LP, US), corresponden a $B$. cordatum, lo que indica la presencia de la especie en las Antillas, aunque no se la haya mencionado previamente.

Algunas especies del paleotrópico tienen fenotipos similares a $B$. cordatum, en particular B. capense (L.) Schltdl. y $B$. procerum (Forst.) Sw. Blechnum capense tiene una distribución que se extendería desde Sudáfrica hasta Polinesia y también es fenotípicamente variable. Chambers \& Farrant (1998) describieron el complejo $B$. procerum para Nueva Zelanda y de ese estudio también se desprenden algunas coincidencias morfológicas entre $B$. 
procerum y B. cordatum, como los contornos y márgenes de las pinnas, el tipo y distribución del indumento, el fenotipo de las etapas de desarrollo y la ornamentación de las esporas. Las medidas esporales del material neocelandés de $B$. procerum $[(45)$ 56 (70) x (25) 39 (52) um] son menores que las registradas para B.cordatum [(60) $62(74)$ x (44) $47(57) \mu \mathrm{m}]$ y los datos citológicos existentes, uno para cada especie, discrepan: Chambers (1954) reportó un número $2 \mathrm{n}=112$ para $B$. procerum de Nueva Zelanda, es decir, sería un tetraploide basado en un número básico $\mathrm{x}=28$, distinto del mencionado antes para el material chileno de B. cordatum.

Si bien Blechnum es un género que incluye especies típicamente americanas y otras exclusivamente paleotropicales, así como endemismos, no puede dejar de considerarse la existencia de taxones con áreas actualmente disyuntas pero que podrían explicarse por una dispersión antigua relacionada con una historia evolutiva relativamente larga.

AGRADECIMIENTOS. Las autoras desean agradecer al Dr. Raúl Perdomo, Vicerrector de la Universidad de La Plata y a la Administración de Parques Nacionales de Argentina (APN), por el apoyo brindado en el curso del desarrollo del proyecto y para los estudios de campo y al Dr. José M. Gabriel y Galán por el análisis de los especímenes del herbario UC.

\section{REFERENCIAS}

ARANA, M., M. PONCE y N. VISCHI -2004Sinopsis de los helechos y grupos relacionados (Pteridophyta) de la provincia de Córdoba, Argentina. Bol. Soc. Argent. Bot. 39 (1-2): 89-114.

CABRERA, A y A WILLINK -1973- Biogeografía de América Latina. Secretaría General de la OEA, Monografía $N^{\circ} 13$, Serie de Biología.

CHAMBERS, T.C. -1954- Experimental studies on the genus Blechnum in New Zealand. Tesis
Doct. inéd. Aukland University College, University of New Zealand.

CHAMBERS, T.C. \& P. A. FARRANT -1998The Blechnum procerum ("capense") complex in New Zealand (Blechnaceae). New Zealand J. Bot. 36: 1-19.

DE LA SOTA, E. R. -1977- Familia Blechnaceae. In: A. L. Cabrera (ed.): Flora de la provincia de Jujuy, Argentina II, Pteridophyta. Colección Científica del INTA, Buenos Aires.

DELUCCHI, G. -2006- Las especies vegetales amenazadas de la provincia de Buenos Aires: una actualización. Boletín Cientifico APRONA XIV (39): 19-31.

DURÁN, M. L. -1997- Estudios morfólogicos, taxonómicos y biosistemáticos en el género Blechnum (Blechnaceae-Pteridophyta). Tesis Doctoral, inéd. Facultad de Ciencias Exactas, Físicas y Naturales, Universidad Nacional de Córdoba, Argentina.

JARA-SEGUEL, P., M. ROMERO-MIERES y C. PALMA-ROJAS -2006- Números cromosómicos de Pteridófitos chilenos: primera contribución. Gayana Bot. 63(1): 115-118.

KASMIRCZAK, C. -1999- A familia Blechnaceae (C. Presl) Copel. (Pteridophyta) no Rio Grande do Sul. Tesis (Grau do Mestre), Universidade Federal do Rio Grande do Sul. Brasil.

LELLINGER, D. B. -2002- A Modern Multilingual Glossary of taxonomic Pteridology. Pteridologia 3: 1- 246.

LELLINGER, D. B. \& W. C. TAYLOR -1997- A classification of spore ornamentation in the Pteridophyta, pp. 33-42. In: R. J. Johns (ed.), Holttum Memorial Volume. Royal Botanic Gardens, Kew.

MICKEL, J. T. \& A. R. SMITH -2004- The Pteridophytes of Mexico. Mem. New York Bot. Gard. 88: 1-1054.

MORAN, R.C. -1995- Blechnaceae. In: G. Davidse, M. Souza, \& S. Knapp, (eds.), Flora Mesoamericana I, Psilotaceae a Salviniaceae. Universidad Autónoma de México, Missouri Botanical Garden \& The Natural History Museum (London).

OLIVEIRA DITTRICH, V. A. -2005-Estudos taxonômicos no gênero Blechnum L. (Pteridophyta-Blechnaceae) para as regiões 
Sudeste e Sul do Brasil. Tesis Doctoral, Universidad Estadual Paulista Julio de Mesquita Filho, Rio Claro, São Paulo, Brasil. PÉREZ-GARCÍA, B., A. MENDOZA y M. RICCI -1996- Morfogénesis de la fase sexual de B. chilense y Blechnum cycadifolium (Pterophyta: Blechnaceae). Rev. Biol. Trop. 44: 491-497.

PONCE, M. M. -1996- Pteridophyta. In: F. O. Zuloaga \& O. Morrone (eds.), Catálogo de las plantas vasculares de la República Argentina, Parte I. Monogr. Syst. Bot. Missouri Bot. Gard. 60: 1-79.

PRABHAKAR, M. -2003- Structure, delimitation, nomenclature and classification of stomata. Acta Botanica Sinica 46 (2): 242252. 2003.

PRADA, C. \& C. H. ROLLERI -2005- A new species of Isoetes L. (Isoetaceae) with a study of microphyll intercellular pectic protuberances and their potential taxonomic value. Bot. J. Linn. Soc. 147 (2): 213-228.

RAMOS GIACOSA, J. P., E. R. DE LA SOTA \& G. E. GIÚDICE -2004- Actualización florística y análisis numérico de la biodiversidad de la pteridofitas de la provincia de Buenos Aires. Bol. Soc. Argent. Bot. 39 (1-2): 125-130.

RAMOS GIACOSA, J. P., E. R. DE LA SOTA \& G. E. GIÚDICE -2006- Blechnum cordatum (Blechnaceae): nueva cita para la flora del noroeste de la Argentina. Bol. Soc. Argent. Bot. 41 (1-2): 91-93.

RODRÍGUEZ-RÍOS, R. -1973- Morfología de los protalos y esporofitos jóvenes de algunas especies chilenas de Blechnum (Polypodiaceae s.1.). Gayana Bot. 22: 1-30.

RODRÍGUEZ RÍOS, R. -1995- Pteridophyta. In: C. Marticorena \& R. Rodríguez R. (eds.), Flora de Chile, I. Ediciones de la Universidad de Concepción, Concepción, Chile.

ROLLERI, C. H. -1984- Ontogenia y semofilesis de las lenticelas de algunas Filicophyta, con especial referencia a las presentes en Blechnum chilense (Kaulf.) Mett., Blechnaceae. Rev. Museo La Plata, Botánica 13 (74): 165-187.

ROLLERI, C. H. \& C. PRADA -2006a- Catálogo comentado de las especies mesoamericanas y sudamericanas de Blechnum L., Blechnaceae-Pteridophyta. Anales Jar. Bot. Madrid 63(1): 67-106.

ROLLERI, C. H. y C. PRADA -2006b- Revisión de los grupos de especies del género Blechnum L. (Blechnaceae-Pteridophyta), el grupo B. penna-marina. Acta Bot. Malacitana 31(1): 7-50.

SANTOS CONDACK, J. P. -2006- Pteridófitas ocorrentes na região alto do Parque Nacional do Itatiaia: análise florística e estructural. Dissertacão (Mestrado). Instituto de Pesquisas Jardim Botânico do Rio de Janeiro. Brasil.

STEYERMARK, J. A., P.A. BERRY, B.K. HOLST \& K. YATSKIEVYCH, (Eds.) -1995Flora of the Venezuelan Guayana, Vol. 2, Pteridophytes and Spermatophytes (Acanthaceae-Araceae). Missouri Botanical Garden/Timber Press, St. Louis/Portland.

STOLZE, R. G. -1981- Ferns and fern allies of Guatemala. part. 2: Polypodiaceae. Fieldiana Botany 6: 1-522.

TRYON, R. M. \& R. G. STOLZE -1993Pteridophyta of Peru. Part. 5: AspleniaceaePolypodiaceae. Fieldiana Bot., n. s. 32: 5470.

TRYON, R. M. \& A. F. TRYON -1982- Ferns and fern-allies from Tropical America. Springer-Verlag, New York. 